\title{
The use of piezosurgery as an alternative method of minimally invasive surgery in the authors' experience
}

\author{
Mansur Rahnama, Łukasz Czupkałło, Leszek Czajkowski, Joanna Grasza, Jan Wallner
}

Chair and Department of Oral Surgery, Medical University of Lublin, Lublin, Poland

\begin{abstract}
Abstarct
Piezosurgery is a relatively new technique of bone surgery that is recently gaining popularity in implantology, periodontics and oral surgery. The piezosurgery device produces specific ultrasound frequency modulation (22 000$35000 \mathrm{~Hz}$ ). The unit provides extreme precision and safety as well as micrometric cutting, thus allowing one to selectively section the mineralized bone structures. Moreover, the device causes less bleeding during and after the operation and the healing process is shorter. Due to the aforementioned advantages, an ultrasound device could be utilized in a wide range of surgical procedures, e.g. impacted tooth extraction, elevation of the Schneiderian membrane, bone splitting or expansion of the ridge, preparing bone bed and bone sampling, and corticotomy, not to menton cystectomy.
\end{abstract}

Key words: piezosurgery, ultrasonic device, osteotomy, alveolar splitting, sinus lifting, tooth extraction.

\section{Introduction}

\section{What is ultrasonics?}

Ultrasonics are vibrations of frequencies greater than the upper limit of the audible range for humans -that is, greater than about $20 \mathrm{kHz}$. The term sonic is applied to ultrasound waves of very high amplitudes [1].

\section{Piezosurgery basics}

It was Pierre Curie who discovered in 1881 piezoelectricity - the phenomenon that gave the basis of piezosurgery, developed in the mid $20^{\text {th }}$ century. Piezoelectricity is found in some crystals that, when subjected to mechanical charges, acquire electric polarization [2]. An ultrasonic transducer is a device used to convert some other type of energy into an ultrasonic vibration. By far the most popular and versatile type of ultrasonic transducer is the piezoelectric crystal, which converts an oscillating electric field applied to the crystal into a mechanical vibration. Piezoelectric crystals include quartz, Rochelle salt, and certain types of ceramic. Piezoelectric transducers are readily employed over the entire frequency range and at all output levels. Particular shapes can be chosen for particular applications. For example, a disc shape provides a plane ultrasonic wave, while curving the radiating surface in a slightly concave or bowl shape creates an ultrasonic wave that will focus at a specific point.

Piezoelectric and magnetostrictive transducers also are employed as ultrasonic receivers, picking up an ultrasonic vibration and converting it into an electrical oscillation $[1,3,4]$.

There is also an inverse piezoelectricity, where crystals are subjected to an electric charge and they acquire a mechanical charge; in case of the electric charge being alternative, the crystals expand and contract alternately, and if on top of that we add an 
intermediate frequency, the crystals produce mid-frequency mechanical oscillations, producing ultrasonic waves. Ultrasonic waves are mechanical waves which, because of the phenomenon of agitation, can induce the disorganization and fragmentation of different bodies. The ultrasonic vibrations can easily allow the segmentation of interfaces from solid to solid by means of distinct vibration, and solid-liquid by means of cavitation. These two concepts are the basis of piezosurgery technology used these days in the dental field $[1,3,4]$.

\section{Ultrasonic devices in medicine}

According to the literature, in 1950 Pohlman was the first to apply ultrasound to human tissues as the treatment of myalgias and neuropathic pain $[3,6]$. The same year Maintz revealed the positive effect on regeneration and healing of the bone [7].

Two years later, in 1952, an ultrasonic unit was used in dentistry for preparation of cavities [3]. Finally in 1988 the ultrasonic phenomenon was applied in the field of oral surgery. But dental surgical techniques have been changed and developed significantly during the last 20 years [8]. In current devices the ultrasonic frequency is modulated by the surgeon from 10, 30 and 60 cycles $/ \mathrm{s}(\mathrm{Hz})$ even up to $35 \mathrm{kHz}$. The low frequency enables cutting of mineralized structures, but not soft tissue. The power ranges from $2.8 \mathrm{~W}$ to $16 \mathrm{~W}$ depending on the various types of bone density. The tip of the ultrasonic devices ranges from $20 \mu \mathrm{m}$ to $200 \mu \mathrm{m}[4,8]$.

Our work with an ultrasonic unit over several years has given us a lot of experience.

\section{Material and methods}

In this article we present 6 different oral surgery procedures during which we used a Surgysonic II piezo device manufactured by Esacrom (Italy). This technique also has usage in cranio-maxillo-facial surgery, orthognathic surgery, oncology and laryngology [9-12]. The ultrasonic unit Surgysonic II consists of a central unit with a digital control panel, a control pedal, and a handpiece with a variety of surgical tips that are designed for different purposes depending on the type of surgical field. The Surgysonic II unit offers 10 programs in each function (surgery, normal and endo). The amplitude of the microvibrations is in the range of 20 to 200 microns with frequency $22000-35000 \mathrm{~Hz}$, corresponding to a handpiece po- wer rating of $50 \mathrm{~W}$. The intensity of the fluid that cools the surgical site can be adjusted in the range from $0 \mathrm{ml} / \mathrm{min}$ to $70 \mathrm{ml} / \mathrm{min}$, optimizing the cooling of the headpieces for different purposes.

\section{Results}

Due to the possibility of switching the ultrasonic tips as well as frequency of the device the usage of the ultrasound unit in oral surgery practice is broad.

\section{Tooth extraction}

One of the advantages of the ultrasonic unit is the ability to prepare the bony window in the external cortex. That provides an easy access to the impacted tooth or root with limited loss of bone [3]. Moreover, it enables one to replace the removed bony piece in its previous position to improve the healing process and reduce the regeneration period (Photos 1-3).

\section{Sinus lift procedure}

Elevating the maxillary sinus membrane is the most successful therapeutic method in the case of the atrophic alveolar ridge in the posterior part of the maxilla. The traditional sinus lift membrane technique implies a great risk of perforating the Schneider membrane, which is the most common complication. It can occur either while separating the sinus membrane or while preparing the window and performing the ostectomy to reach the sinus membrane using the surgical round bit of the piece of low speed (Photo 4) $[4,10]$.

\section{Cystectomy}

This technique does not require one to cut soft tissues (gingivae, vessels, nerves, muscles). Therefore the cystectomy can be performed without risk of injuring the adjacent underlying nerves or vessels (Photos 5, 6) [4, 9].

\section{Bone splitting}

During implantology treatment in case of insufficient width of the alveolar ridge the splitting technique can be applied. In this field piezosurgery technology provides great effects. The osteotomy tips allows an atraumatic bone separation. The separating can be finished with manual osteotomes. The ultrasonic unit decreases the risk of breaking the bone 


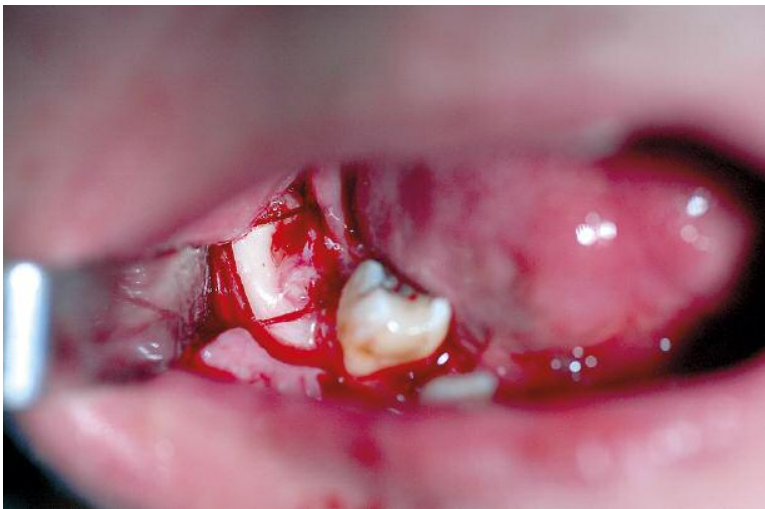

Photo 1. Piezosurgery allows one to extract the impacted tooth atraumatically

owing to expansion of the bone; thus it tends to become more elastic (Photos 7-9) [9, 12].

\section{Bone osteotomy or corticotomy}

Bone corticotomy and distraction of the periosteum allow one to dislocate impacted or semiimpacted teeth in a shorter period of time. It can concern either single or multiple teeth during orthodontic treatment (Photo 10) [12].

\section{Harvesting bone blocks and bone grafting}

The ultrasound unit gives the possibility to collect autologous grafts in the form of monocortical blocks or bone chips. Bone chips as big as $500 \mu \mathrm{m}$ are the basis of osteoconductive bone regeneration [12]. The piezo unit enables one to gently scrub the bone surface to gain the right amount of graft material. As far as bone blocks are concerned, the donor beds could be situated in the chin, oblique line of the mandible or iliac crest. Using this technique the easiness of the surgical procedure as well as smaller entrance site are guaranteed (Photo 11) [10, 13].

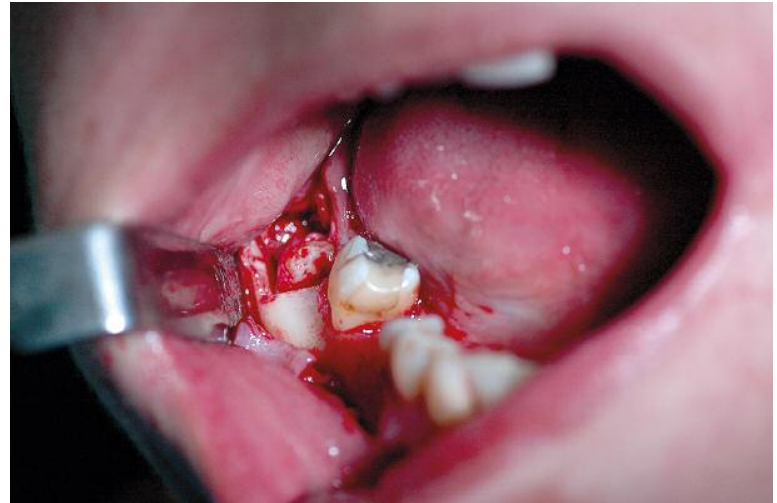

Photo 2. Preparation of the bony window in the external cortex that provides an easy access to the impacted tooth with limited loss of bone

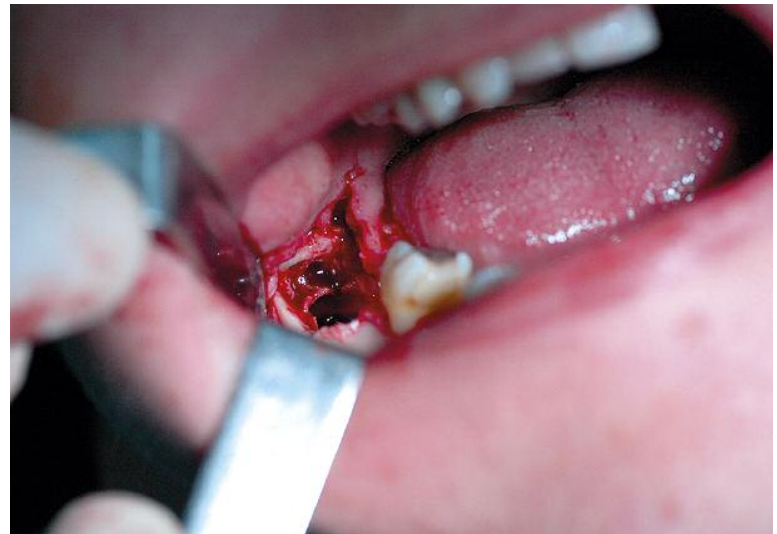

Photo 3. Extraction site of impacted third molar ready to be replaced with removed bony piece

\section{Discussion}

Piezoelectric surgery applied to bone presents a real alternative method of minimally invasive surgery. In comparison with the traditional method it offers a massive advantage. Nevertheless, this technique requires special surgical skill and involves
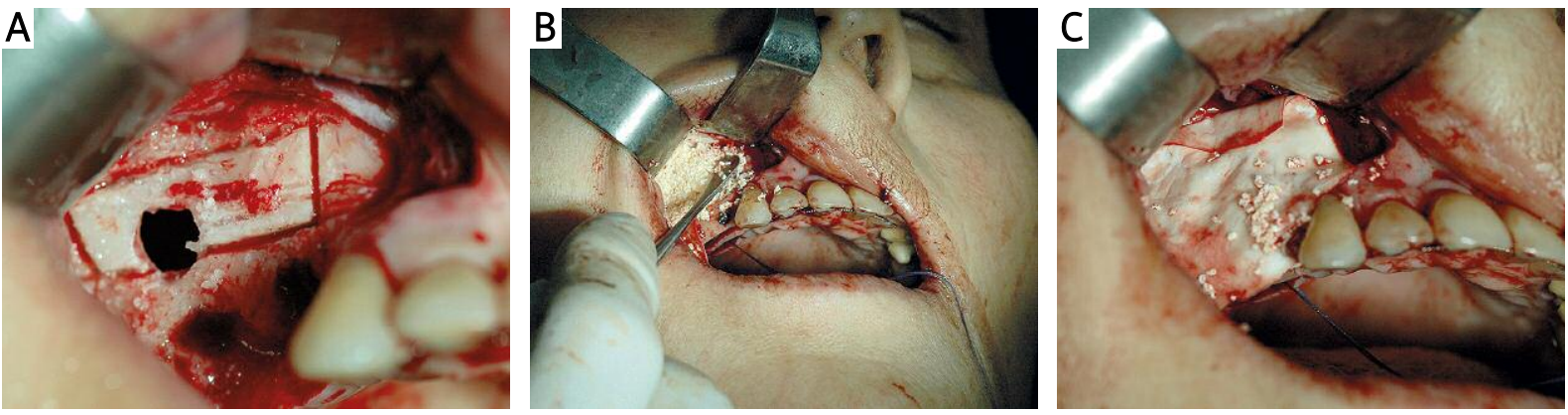

Photo 4 A-C. Membrane elevation of the maxillary sinus and bone graft procedure 


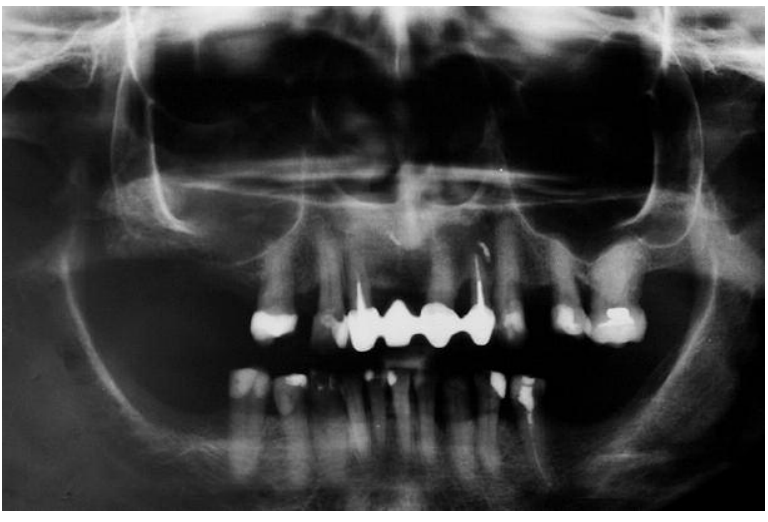

Photo 5. Panoramic X-ray showing the cyst of the right sinus cavity

a certain knowledge. It is an innovation in bone surgery and the characteristics differ from those of the conventional technique.

\section{Advantages over conventional surgical equipment}

\section{Precise cutting and safety}

The ultrasonic device enables micrometric cutting that depends on the micro-oscillation of the hand- piece. It varies from $20 \mu \mathrm{m}$ to $200 \mu \mathrm{m}$, which is smaller than the width achievable with rotary instruments. Therefore it offers superior precision in cutting with no bone loss [3].

\section{Great control of the surgical device}

To achieve the desired efficiency, conventional drills must be loaded with a force of $2 \mathrm{~kg}$ to $3 \mathrm{~kg}$, whereas the piezosurgical handpiece reaches its highest efficiency when applying a load of $0.5 \mathrm{~kg}[10,14-16]$.

\section{Bleeding-free surgery site}

Cutting with the ultrasound unit does not cause bleeding of bone tissue, and thus provides good visibility of the operating site and enables one to conduct the procedure very precisely. The reason for this is that the cavitation effect creates bubbles from the physiological salt solution and these lead to implosion and generate the shock wave causing microcoagulation [14-17].

\section{Selective cutting and minimal operative invasion}

It reduces the risk of perforating the Schneider membrane due to the selective cutting that is limited only to the mineralized structures - bone. This is
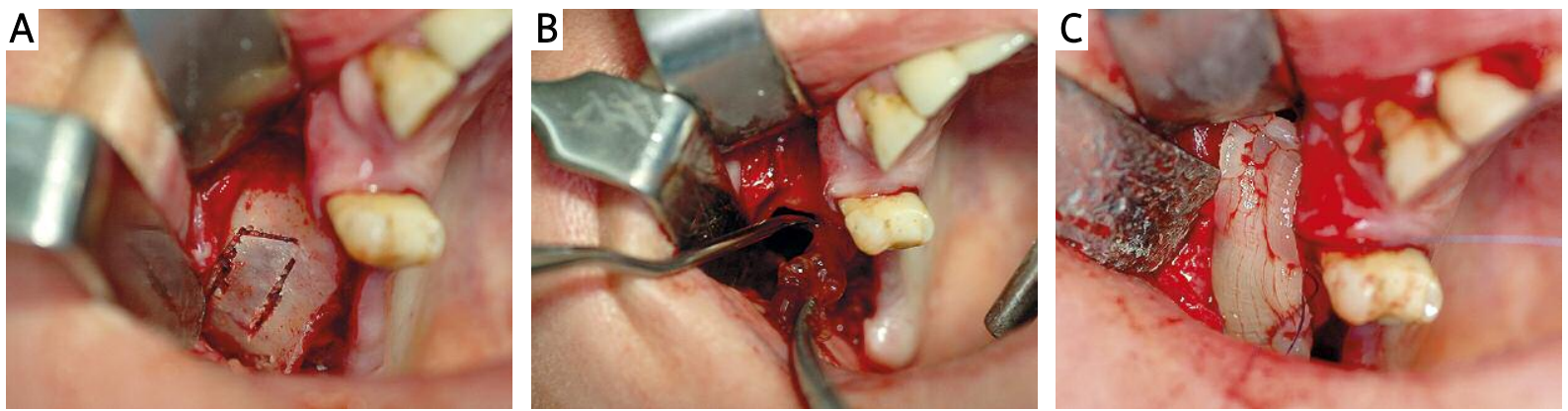

Photo 6 A-C. Minimal operative and atraumatic cystectomy

A

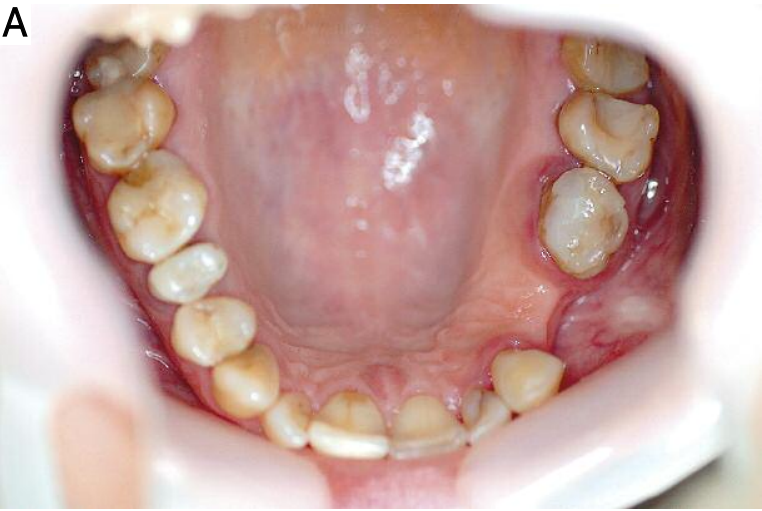

Photo 7 A, B. Clinical view showing the bone defect in the zone of 1.2

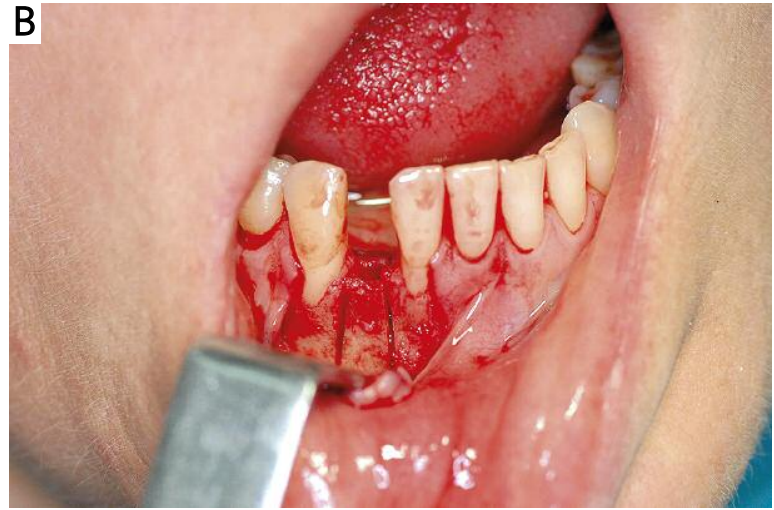




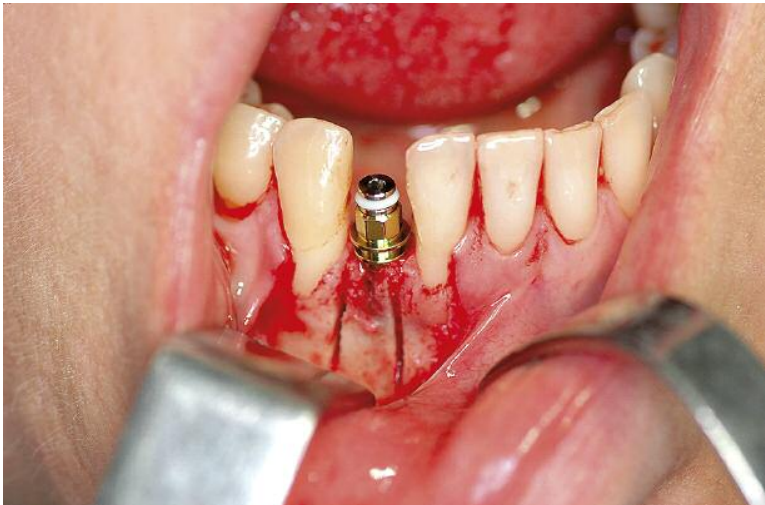

Photo 8. Bone splitting during surgical procedure

because of the ultrasonic frequencies that are used $(25-29 \mathrm{kHz})$, as the hard tissues and soft tissues are cut at a different frequency [10].

\section{Faster bone regeneration and healing process}

Oxygen molecules released during cutting have an antiseptic effect and ultrasound vibration stimulates cells' metabolism. Moreover, the lack of necro-

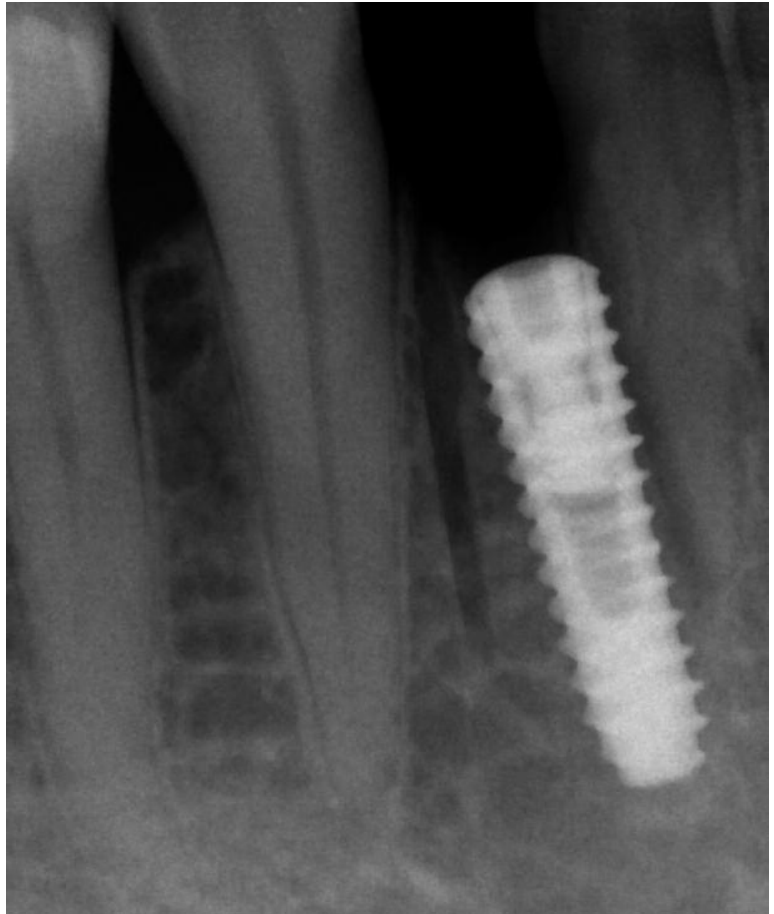

Photo 9. Effectively split bone and implantation
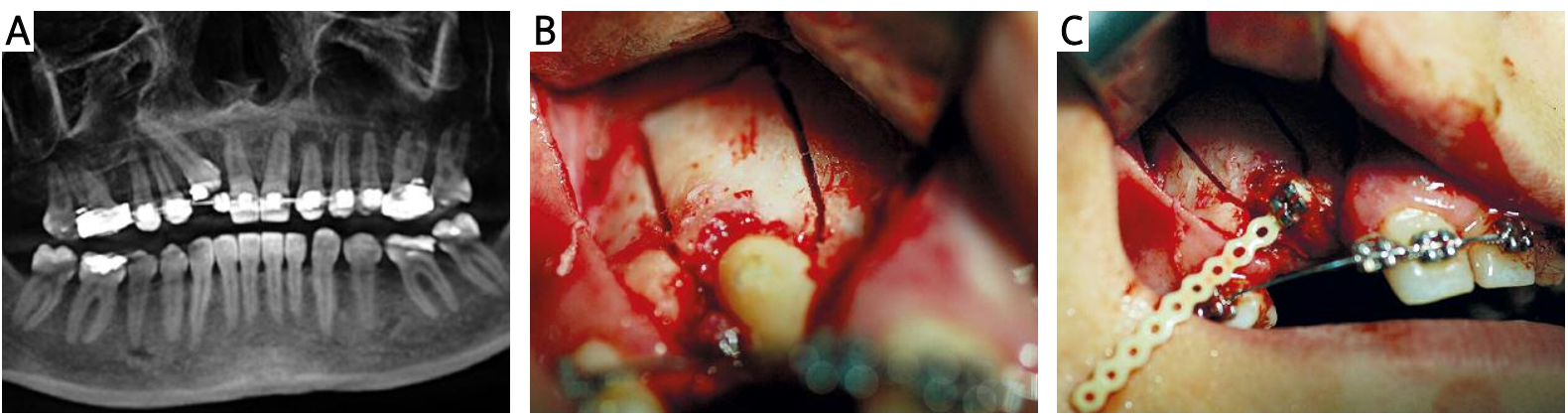

Photo $10 \mathrm{~A}-\mathrm{C}$. Piezosurgery can accelerate orthodontic treatment in dislocating impacted teeth in a shorter period of time
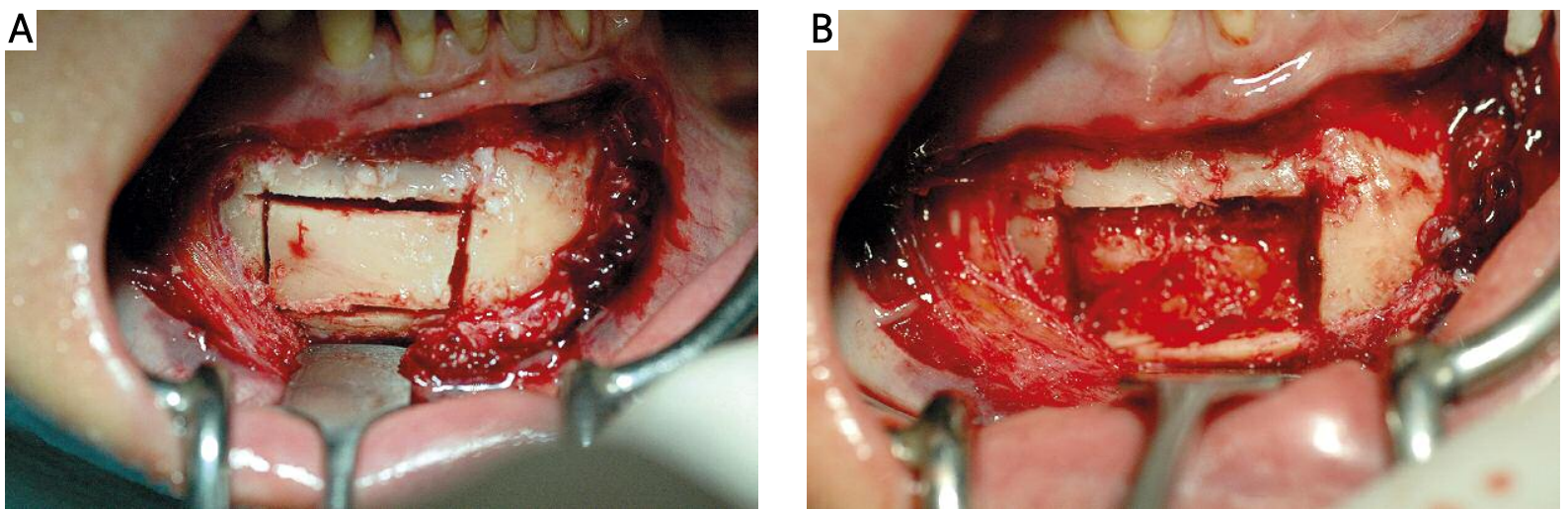

Photo 11 A, B. Harvesting of the chin graft 
sis in the cut area accelerates bone regeneration. Soft tissue damage is not noticed [10, 14-16].

\section{No risk of emphysema}

The risk of subcutaneous emphysema is reduced due to the aerosol effect that the ultrasonic device produces unlike the effect of air-water spray generated be osteotomy with rotary instruments $[4,13]$.

\section{Decrease in post-intervention pain}

Owing to the action being less invasive and producing less collateral tissue damage, it results in better healing $[3,8]$.

\section{Reduced traumatic stress}

The device produces less noise and only microvibrations in comparison with a conventional motor, so the fear and psychological stress of the patient are reduced [14].

\section{Disadvantages and precautions}

The largest disadvantage of the procedure using the piezosurgery unit is the increased operation time that is required for bone preparation.

The piezosurgery technique immensely lessens the risk of damaging soft tissues, such as sinus floor membrane, nerves, and vessels, but nevertheless precautions must be taken as the ultrasonic waves have mechanical energy, and this energy can be converted into heat and pass into adjacent tissues. For this reason the use of irrigation is essential, not only for the effect of cavitation, but also to avoid overheating [14-16]. The intensity of the cooling liquid can be adjusted depending on different preparations.

\section{Conclusions}

According to personal experience and other authors' experience, it can be truly said that during some surgical procedures the piezo device provides a great facility and even to some extent becomes indispensable. The ultrasound unit allows for precise removal of bone with minimal risk of injury to underlying soft tissues. It allows a more successful and more complication-free surgical result for a less experienced surgeon that could be especially beneficial for preparation of the Schneider membrane during sinus lift procedure or relocating or preparation of the inferior alveolar nerve [18]. Not only does it give minimal operative invasion, but also it decreases post-inter- vention pain and reduces traumatic stress, not to mention less bleeding intra- and postoperatively [3].

\section{References}

1. Ultrasonics. Encyclopædia Britannica. Encyclopædia Britannica Online. Encyclopædia Britannica Inc., 2012. Web. 09 Sep. 2012 http://www.britannica.com/EBchecked/topic/613488/ultrasonics.

2. Biesaga Ł, Grzesiak-Janas G, Janas A. Piezoelectric surgery. Por Stomatol 2010; 10: 353-5.

3. Escoda-Francolí J, Rodríguez-Rodríguez A, Berini-Aytés L, et al. Application of ultrasound in bone surgery: two case reports. Med Oral Patol Oral Cir Bucal 2010; 15: 902-5.

4. Vercellotti T. Technological characteristics and clinical indications of piezoelectric bone surgery. Minerva Stomatologica 2004; 53: 5.

5. Vercellotti T. Caracteristicas technologicas e indicationes clinicas de la chirurgia osea piezoelectrica. Revista Mundo Dental 2005; 26-8

6. Kennedy JE, Ter Haar GR, Cranston D. High intensity focused ultrasound: surgery of the future? Br J Radiol 2003; 76: 590-9.

7. Maintz G. Animal experiments in the study of the effect of ultrasonic waves on bone regeneration. Strahlentherapie 1950; 82: 631-8.

8. Hema S, Kranti K, Sameer Z. Piezosurgery in periodontology and oral implantology. J Indian Soc Periodontol 2009; 13: 155-6.

9. Stupka M. Use of piezosurgery in treatment of jaw cysts. Implantoprotetyka 2010; 2-3: 56-61.

10. Schlee M, Steigmann M, Bratu E, et al. Piezosurgery: basics and possibilities. Implant Dent 2006; 12: 334-40.

11. Labanca M, Azzola F, Vinci R, et al. Piezoelectric surgery: twenty years of use. Br J Oral Maxillofac Surg 2008; 4: 265-9.

12. Happe A. Use of a piezoelectric device to harvest bone grafts from the mandibular ramus: case reprt of 40 cases. Int J Periodontics Dent 2007; 7: 241-9.

13. Sohn DS, Ahn MR, Lee WH, et al. Piezoelectric osteotomy of intraoral harvesting of bone blocks. Int J Periodontics Restorative Dent 2007; 27: 3-7.

14. Stübinger S, Landes C, Seitz O, et al. Ultrasonic bone cutting in oral surgery: a review of 60 cases Ultraschall Med 2008; 29: 66-71.

15. Torrella F, Pitarch J, Cabanes G, et al. Ultrasonic osteotomy maxillary sinus: a technical note. Int J Oral Maxillofac Implants 1998; 13: 697-700.

16. Troiani C, Russo C, Ballarani G, et al. Piezoelectric surgery: a new reality to cut and manage bone in maxillo-odonto-stomatology. Int J Maxillo Odontostomatology 2005; 4: 23-8.

17. Metzger MC, Bormann KH, Schoen R, et al. Inferior alveolar nerve transposition - an in vitro comparison between piezosurgery and conventional bur use. J Oral Implantol 2006; 32: 19-25.

18. Wallace SS, Mazor Z, Froum SJ, et al. Schneiderian membrane perforation rate during sinus elevation using piezosurgery: clinical results of 100 consecutive cases. Int J Periodontics Restorative Dent 2007; 27: 5.

Received: 14.01.2013, accepted: 28.04.2013. 